\title{
Management of the anticoagulated trauma patient in the emergency department: a survey of current practice in England and Wales
}

\author{
Ceri E Battle, ${ }^{1}$ Fiona E Lecky, ${ }^{2}$ Tom Stacey, ${ }^{3}$ Antoinette Edwards, ${ }^{3}$ Phillip A Evans ${ }^{4}$
}

\begin{abstract}
- Additional material is published online only. To view please visit the journal online (http://dx.doi.org/10.1136/ emermed-2015-205120).

${ }^{1}$ NISCHR Haemostasis Biomedical Research Unit Epidemiology Division, Morriston Hospital, Swansea, UK

${ }^{2}$ The Trauma Audit and Research Network, University of Manchester, University of Sheffield/University of Manchester/Salford Royal Hospital NHS Foundation Trust. Emergency Medicine Research in Sheffield (EMRiS), Health Services Research, School of Health and Related Research, Sheffield, UK

${ }^{3}$ The Trauma Audit and Research Network, University of Manchester, Salford, UK

${ }^{4}$ NISCHR Haemostasis Biomedical Research Unit, Morriston Hospital, Swansea, UK
\end{abstract}

\section{Correspondence to} Dr Ceri Battle, Physiotherapy Department, Morriston Hospital, Morriston, Swansea SA6 6NL, UK

Ceri.battle@wales.nhs.uk

Received 15 June 2015 Revised 30 November 2015 Accepted 13 December 2015 Published Online First

4 January 2016

CrossMark

To cite: Battle CE, Lecky FE, Stacey T, et al. Emerg Med $J$ 2016;33:403-407.

\section{ABSTRACT}

Objective The aim of this study was to investigate current management of the anticoagulated trauma patient in the emergency departments (EDs) in England and Wales.

Methods A survey exploring management strategies for anticoagulated trauma patients presenting to the ED was developed with two patient scenarios concerning assessment of coagulation status, reversal of international normalised ratio (INR), management of hypotension and management strategies for each patient. Numerical data are presented as percentages of total respondents to that particular question.

Results 106 respondents from 166 hospitals replied to the survey, with $24 \%$ of respondents working in a major trauma unit with a specialist neurosurgical unit.

Variation was reported in the assessment and management strategies of the elderly anticoagulated poly-trauma patient described in scenario one. Variation was also evident in the responses between the neurosurgical and non-neurosurgical units for the headinjured, anticoagulated trauma patient in scenario two.

Conclusion The results of this study highlight the similarities and variation in the management strategies used in the EDs in England and Wales for the elderly, anticoagulated trauma patient. The variations in practice reported may be due to the differences evident in the available guidelines for these patients.

\section{INTRODUCTION}

Major trauma accounts for over 5 million deaths worldwide each year and this number is predicted to increase to over 8 million by $2010 .^{1}$ In the UK alone, trauma accounts for approximately 16000 deaths and costs the NHS between $£ 0.3$ and $£ 0.4$ billion in immediate treatment. ${ }^{2}{ }^{3}$ It has been reported that approximately one-third of bleeding trauma patients present with a coagulopathy. ${ }^{1} 4$ Research has demonstrated that coagulation defects that occur in trauma patients are complex and these abnormalities are caused by a number of inter-related factors including pre-existing conditions, pre-injury oral anticoagulant use, dilution of haemostatic factors by fluid resuscitation or blood transfusion, severe hypothermia, hypoperfusion and acidosis due to tissue damage from trauma. ${ }^{5-7}$

It is reported that approximately $1 \%$ of the UK population are currently using anticoagulant therapy and it is predicted that this figure will continue to rise as the size of the elderly population increases. ${ }^{8}$ Controversy exists in trauma research regarding the impact of pre-injury anticoagulant

\section{Key messages}

What is already known on this subject As a result of the conflicting evidence regarding the effect of pre-injury warfarin use on trauma patients, there is a lack of consensus regarding the optimal management strategies for this patient group. As a result, there are no internationally accepted, fully validated guidelines outlining the optimal management strategies for this patient group.

\section{What might this study add?}

This study reports the similarities and variations in current management strategies used for the management of the elderly, anticoagulated trauma patient in England and Wales.

use on trauma patients. Pre-injury warfarin has been reported to be an independent predictor of mortality in trauma patients. ${ }^{9} 10$

There is conflicting evidence regarding the effect of pre-injury warfarin and as a result, no internationally accepted, validated guidelines exist for the management of this group..$^{610-12}$ One of the key recommendations in the National Audit Office Report 'Major Trauma Care in England' (2010) stated that there is a need for Strategic Health Authorities to develop protocols for the effective delivery of major trauma care against the standards set out in national clinical guidelines. ${ }^{13}$ In the absence of such guidelines, there is a risk that patients receive a standard of care that is 'less than good standard', as previously reported in $60 \%$ of major trauma cases in England. ${ }^{14}$ The aim of this study was to investigate variation in current management of the anticoagulated trauma patient in the emergency departments (EDs) in England and Wales, thus highlighting the need and providing evidence for inclusion of this complex subgroup of patients in future national trauma guidelines.

\section{METHODS}

A survey exploring management strategies for anticoagulated trauma patients presenting to the ED was developed. Two patient scenarios were presented, which included a combination of closed and openended questions, with space provided to offer comments as required. A number of questions were open-ended in order not to lead the respondent 


\section{Scenario one}

Scenario 1: A poly-trauma patient has been admitted to your ED. The patient is 70 years old and known to use warfarin for atrial fibrillation. On initial examination, the patient appears to have a closed abdominal injury with signs of shock. The patient has a respiratory rate of 25 , heart rate of 130 , blood pressure (BP) of 90/70 and a normal Glasgow coma scale (GCS). International normalised ratio (INR) is 2.8. The patient has received $1 \mathrm{~L}$ of normal saline during pre-hospital care. Assuming the trauma team is giving tranexamic acid and initiating imaging requests:

1) How would you assess this patient's coagulation status?

2) Would you reverse the patient's INR? YES/NO (Please circle)

Comments:

3) If yes, how would you reverse the INR? (Please circle all applicable)
a) Vitamin K
b) Fresh frozen plasma (FFP)
c) Prothrombin complements

Comments:

4) What INR range are you aiming to achieve through reversal?

5) Would you treat the patient's hypotension? YES/NO (Please circle)

6a) If yes, what would be your initial treatment for the hypotension?

Type of treatment: Volume: Rate of infusion:

$6 \mathrm{~b})$ What is your target BP?

6c) If yes, what would be your ongoing treatment for the hypotension?

Type of treatment: Volume:

7) Would you treat the hypotension differently due to the pre-injury warfarin use (compared with a patient not using warfarin pre-injury)? YES/NO (Please circle)

Comments

into providing specific responses and to reduce the risk of introducing response bias. The survey was pretested by a number of non-participating clinicians in Morriston Hospital, Swansea, and was subsequently revised based on feedback received.

Using a purposive sample, the survey was administered using a web-based form to one of the trauma leads in each of the hospitals participating in the Trauma Audit and Research Network (TARN) in England and Wales. The respondents were therefore asked to discuss their own practice in the survey. To improve initial response rate, a follow-up round of surveys was sent to the trauma leads after 3 months via email and repeated after another 8 weeks. TARN currently receives patient data from all trauma-receiving hospitals in England and Wales. Ethical approval was not required as all responses were anonymous.

Numerical data are presented as percentages of total respondents to that particular question. Results are reported comparing responses for the neurosurgical centres (major trauma units) with the non-neurosurgical centres (non-major trauma centres) for both scenarios.

\section{RESULTS}

\section{Characteristics of respondents}

A total of 106 respondents from 166 hospitals replied to the survey. This was a $64 \%$ response rate from the hospitals participating in TARN. Of these hospitals, 25 out of the 27 neurosurgical centres in England and Wales responded, with the remainder being from non-neurosurgical centres. Six of the included questionnaires had not completed scenario two.

Table 1 outlines how the respondents would assess the patient's coagulation status.

A total of 105 respondents (99\%) stated that they would reverse the patient's INR, demonstrating a consensus in

Table 1 Methods used to assess patient's coagulation status

\begin{tabular}{|c|c|c|c|}
\hline Method of assessment & Number $(\%) n=102$ & $\begin{array}{l}\text { Neurosurgical unit } \\
\text { number }(\%) n=25\end{array}$ & $\begin{array}{l}\text { Non-neurosurgical unit } \\
\text { number }(\%) n=77\end{array}$ \\
\hline INR/near-patient INR & $67(65.7 \%)$ & $12(48 \%)$ & $55(71 \%)$ \\
\hline Coagulation/clotting screen & $48(47.1 \%)$ & $9(36 \%)$ & $39(51 \%)$ \\
\hline Liver function tests & $3(2.9 \%)$ & $1(4 \%)$ & $2(3 \%)$ \\
\hline Laboratory tests (not specified) & $8(7.8 \%)$ & $2(8 \%)$ & $6(8 \%)$ \\
\hline ROTEM or TEG & $11(10.8 \%)$ & $7(28 \%)$ & $4(5 \%)$ \\
\hline Full blood count & $15(14.7 \%)$ & $3(12 \%)$ & $12(16 \%)$ \\
\hline Clinical findings & $8(7.8 \%)$ & $2(8 \%)$ & $6(8 \%)$ \\
\hline Platelets & $6(5.9 \%)$ & $2(8 \%)$ & $4(5 \%)$ \\
\hline Fibrinogen/fibrin degradation product & $16(15.7 \%)$ & $3(12 \%)$ & $13(17 \%)$ \\
\hline Activated partial thromboplastin time & $16(15.7 \%)$ & $2(8 \%)$ & $14(18 \%)$ \\
\hline Prothrombin time & $6(5.9 \%)$ & $2(8 \%)$ & $4(5 \%)$ \\
\hline Other & $6(5.9 \%)$ & $4(16 \%)$ & $2(3 \%)$ \\
\hline Liaise with haematology & $2(2.0 \%)$ & $0(0 \%)$ & $2(3 \%)$ \\
\hline
\end{tabular}

Other: haemoglobin, thrombin time, intracellular calcium, arterial and venous blood gases, D Dimer and Urea and Electrolytes (U\&Es).

Free text box is used and so any number of responses is permitted. Total respondents $=102$.

INR, International normalised ratio; ROTEM, rotational thromboelastometry; TEG, thromboelastography. 


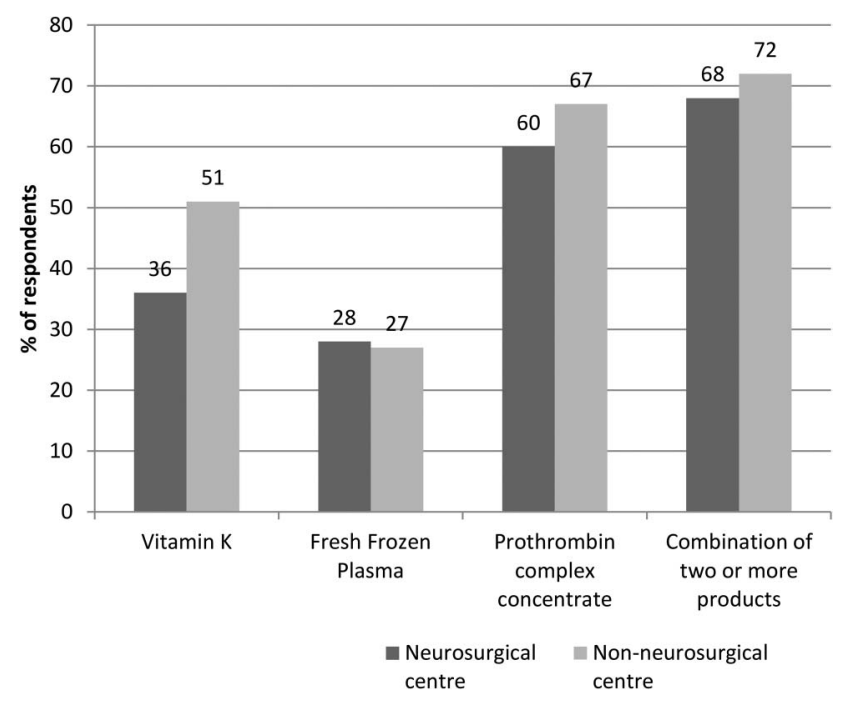

Figure 1 Scenario 1. Products used to reverse the patient's International normalised ratio. Respondents asked to tick which of the three products listed they would use (more than one response therefore permitted).

management between the respondents from both neurosurgical and non-neurosurgical centres. Figure 1 highlights the products used by the respondents to reverse the patient's INR. Respondents may have used either one or any combination of the products.

For the target INR range that respondents were aiming to achieve with their reversal strategies, a total of 21 different values/ranges of values were reported by the respondents, all ranging between the values of 1 and 2 . The most commonly reported target INR (32\%) in the neurosurgical centres was a 'normal' INR, compared with the non-neurosurgical units where the most commonly reported value $(23 \%)$ was 1.5 or less.

A total of $53 \%$ of respondents from the non-neurosurgical units stated they would treat the patient's hypotension, compared with $80 \%$ of respondents from neurosurgical centres. The strategies used by the respondents for the ongoing treatment of the patient's hypotension have been grouped into 'blood products' (including red cells, platelets, FFP and cryoprecipitate) or 'fluids' (including crystalloids, normal saline, Plasma-Lyte and Hartmann's). Table 2 highlights the treatment strategy for ongoing hypotension in the patient in Scenario 1.

A total of $31 \%$ of respondents (from both neurosurgical and non-neurosurgical centres) stressed that the most important
Table 2 Treatment strategies for ongoing hypotension (if hypotension being treated)

\begin{tabular}{|c|c|c|c|}
\hline & $\begin{array}{l}\text { Number (\%) } \\
\mathrm{n}=95\end{array}$ & $\begin{array}{l}\text { Neurosurgical } \\
\text { unit, number } \\
(\%) n=18\end{array}$ & $\begin{array}{l}\text { Non-neurosurgical } \\
\text { unit, number (\%) } \\
\mathrm{n}=77\end{array}$ \\
\hline Blood products & $83(87 \%)$ & $14(78 \%)$ & $69(90 \%)$ \\
\hline Fluids & $23(24 \%)$ & $2(11 \%)$ & $21(27 \%)$ \\
\hline $\begin{array}{l}\text { Massive haemorrhage } \\
\text { protocol }\end{array}$ & $5(5 \%)$ & $2(11 \%)$ & $3(4 \%)$ \\
\hline
\end{tabular}

definitive treatment would be early CT imaging and surgery to identify and address the bleeding source. The volume and rate of each of the products that would be given by the respondents to treat the patient's hypotension are described in online supplementary appendix A.

A total of $58 \%$ of respondents stated their target systolic BP would be between 90 and 100,32\% stressed that they would not use BP at all to assess the patient and 7\% stated that it would depend on whether the patient had a head injury or not. This was the general consensus from respondents in both neurosurgical and non-neurosurgical centres.

A total of $32 \%$ of respondents from the non-neurosurgical centres stated that they would manage this trauma patient differently (compared with other trauma patients) as a result of their pre-injury anticoagulant use. In the neurosurgical centres, a total of $44 \%$ of respondents stated they would manage the patient differently. The main comments made by respondents from both neurosurgical and non-neurosurgical centres regarding the difference in management concerned the need for early reversal of the INR, the need for a higher threshold for suspicion of bleeding, in addition to greater aggressiveness in the correction of clotting abnormalities in the anticoagulated trauma patient.

A total of 100 responses (60\% response rate) were received for the second scenario, with $25 \%$ of the respondents working in a specialist neurosurgical unit. Table 3 highlights the guidelines that would be followed by respondents when managing the patient.

Table 4 highlights the methods the respondents would use to assess the patient's coagulation status. There was a consensus between the two groups that a coagulation screen was the best method for assessing the patient's coagulation status; however, a greater number of respondents from the non-neurosurgical centres relied on INR. Fibrinogen was reported to be used in a higher percentage of respondents from the neurosurgical centres.

\section{Scenario two}

Scenario 2: An elderly patient with an isolated head injury has been admitted to your ED. On examination, the patient is normotensive and has no clinical signs of haemorrhage. The patient is known to use warfarin for atrial fibrillation and has an INR of 2.8. The patient has a GCS of 8 . The patient has a small frontal contusion and a subdural haematoma on CT scan. Assuming the trauma team is considering CRASH 3:

1) Is your ED in a hospital with a specialist neurosurgical unit? YES/NO (please circle)

2) What guidelines would you use to treat this patient?

3) How would you assess this patient's coagulation status?

4) Would you reverse the patient's INR? YES/NO (Please circle)

Comments:

5) If yes, how would you reverse the INR? (Please circle all applicable)

a) Vitamin $\mathrm{K}$

b) FFP

c) Prothrombin complements

Comments:

6) What INR range are you aiming to achieve through reversal? 
Table 3 Guidelines used in the management of anticoagulated trauma patient

\begin{tabular}{lccc}
\hline Guideline & $\begin{array}{l}\text { Number (\%) } \\
\mathbf{n}=\mathbf{9 4}\end{array}$ & $\begin{array}{l}\text { Neurosurgical unit } \\
\text { number (\%) } \mathbf{n = 2 4}\end{array}$ & $\begin{array}{c}\text { Non-neurosurgical } \\
\text { unit number (\%) } \mathbf{n = 7 0}\end{array}$ \\
\hline NICE Head Injury Guidelines & $33(35 \%)$ & $8(33 \%)$ & $25(36 \%)$ \\
Local Trust Guidelines & $30(32 \%)$ & $10(42 \%)$ & $20(29 \%)$ \\
Advanced Trauma Life Support & $3(3 \%)$ & $0(0 \%)$ & $3(4 \%)$ \\
CRASH-3 on Tranexamic Acid & $4(4 \%)$ & $0(0 \%)$ & $4(6 \%)$ \\
British Society of Haematology & $7(7 \%)$ & $2(8 \%)$ & $5(7 \%)$ \\
Regional Trauma or neurosurgical & $12(13 \%)$ & $2(8 \%)$ & $10(14 \%)$ \\
None needed & $5(5 \%)$ & $0(0 \%)$ & $5(7 \%)$ \\
On-call neurologist & $7(7 \%)$ & $1(4 \%)$ & $6(9 \%)$ \\
Other & $5(5 \%)$ & $0(0 \%)$ & $5(7 \%)$ \\
\hline
\end{tabular}

Free text box is used and so any number of responses is permitted.

CRASH-3, Clinical randomisation of antifibrinolytic in significant head injury (trial acronym).

A total of $97 \%$ of respondents stated that they would reverse the patient's INR in this scenario, with the $3 \%$ who would not reverse it, working in a non-neurosurgical centre. Figure 2 highlights the products used by the respondents to reverse the patient's INR in scenario two.

The INR value or range that respondents were aiming to achieve for the patient in scenario two with their reversal strategies ranged between 1 and 2, with a total of 19 different responses given. The most commonly reported target INR value (33\%) for the neurosurgical centres was an INR of 1, compared with the non-neurosurgical centres where the most commonly reported value $(20 \%)$ was 1.5 or less.

\section{DISCUSSION}

This study has identified the similarities and differences in treatment strategies used in England and Wales, to manage the elderly, anticoagulated trauma patient presenting to the ED. Hanley commented that pronounced differences exist in the recommendations within guidelines regarding management of an elderly, anticoagulated trauma patient. ${ }^{15}$ The results of this study have highlighted that a number of different guidelines are used by the respondents in current practice. Furthermore, a number of respondents who work at non-neurosurgical centres suggested that they did not use any guidelines to manage such patients.
The most commonly used guideline (NICE Head Injury Guidelines) only discusses the use of CT scan in this specific patient group and contains no recommendation regarding reversal of anticoagulant therapy. ${ }^{16}$ The British Society of Haematology guidelines recommends the use of intravenous or oral vitamin $\mathrm{K}$ in combination with Prothrombin Complex Concentrate (PCC) or FFP (if PCC not available) for the reversal of warfarin in the bleeding major trauma patient. ${ }^{17}$ It is beyond the scope of this study to comment on similarities or variations in the content of each individual regional or local guidelines. It is evident however that there are no national guidelines that focus solely on the elderly, anticoagulated trauma patient that consider all aspects of management.

When considering both scenarios presented in the survey, there was an overall consensus that the patients' INR should be reversed. The methods used to assess the patient's coagulation status varied between the respondents. The most commonly used methods to assess coagulation status were INR and a clotting/coagulation screen, in both neurosurgical and nonneurosurgical centres. Recent research has suggested however that these tests monitor only the initiation phase of blood coagulation therefore it is possible that the conventional coagulation screen appears normal, while the overall state of blood coagulation (clot stability, lysis or platelet function) is

Table 4 Methods used to assess patient's coagulation status

\begin{tabular}{|c|c|c|c|}
\hline Method of assessment & $\begin{array}{l}\text { Number of } \\
\text { responses }(\%) n=98\end{array}$ & $\begin{array}{l}\text { Neurosurgical } \\
\text { unit number }(\%) n=25\end{array}$ & $\begin{array}{l}\text { Non-neurosurgical } \\
\text { unit number }(\%) n=73\end{array}$ \\
\hline INR/near patient INR & $57(58 \%)$ & $10(40 \%)$ & $47(64 \%)$ \\
\hline Coagulation screen & $46(47 \%)$ & $12(48 \%)$ & $34(47 \%)$ \\
\hline Liver function tests & $4(4 \%)$ & $2(8 \%)$ & $2(3 \%)$ \\
\hline ROTEM or TEG & $4(4 \%)$ & $2(8 \%)$ & $2(3 \%)$ \\
\hline Full blood count & $8(8 \%)$ & $2(8 \%)$ & $6(8 \%)$ \\
\hline Clinical findings & $2(2 \%)$ & $2(8 \%)$ & $0(0 \%)$ \\
\hline Platelets & $7(7 \%)$ & $1(4 \%)$ & $6(8 \%)$ \\
\hline Fibrinogen/fibrin degradation product & $10(10 \%)$ & $7(28 \%)$ & $2(3 \%)$ \\
\hline Activated partial thromboplastin time & $11(11 \%)$ & $1(4 \%)$ & $10(14 \%)$ \\
\hline Partial Prothrombin time & $2(2 \%)$ & $0(0 \%)$ & $2(3 \%)$ \\
\hline Prothrombin Time & $6(6 \%)$ & $2(8 \%)$ & $4(5 \%)$ \\
\hline Other & $4(4 \%)$ & $1(4 \%)$ & $3(4 \%)$ \\
\hline No further assessment required & $8(8 \%)$ & $4(16 \%)$ & $4(5 \%)$ \\
\hline
\end{tabular}

Other: haemoglobin, liaise with the on-call haematologist, intracellular calcium and arterial/venous blood gases.

Free text box used so any number of responses permitted. Total respondents=98.

INR, International normalised ratio; ROTEM, rotational thromboelastometry; TEG, thromboelastography. 


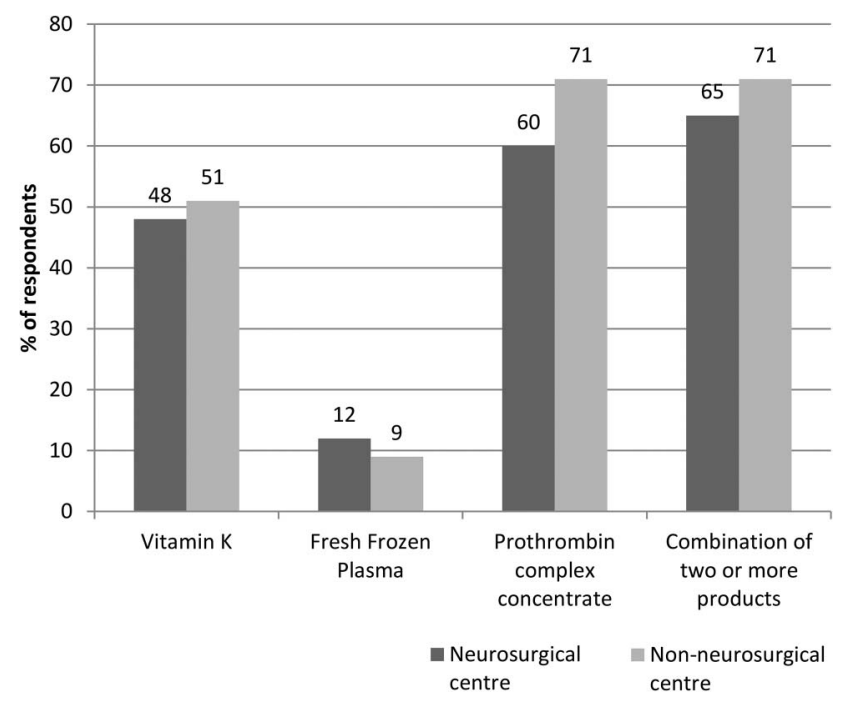

Figure 2 Scenario 2. Products used to reverse the patient's International normalised ratio. Respondents asked to tick which of the three products listed they would use (more than one response therefore permitted).

abnormal. ${ }^{4}{ }^{18}$ It is suggested that in addition to routine coagulation tests, viscoelastic methods must also be performed to assist in characterising the coagulopathy and in guiding haemostatic therapy; however, further research is needed. ${ }^{4}{ }^{18} \mathrm{~A}$ number of respondents commented that they would like to use such methods, but the equipment was not available at their ED. This lack of access to equipment invariably contributes to obvious differences in management of this patient group.

The products used to reverse the patient's INR varied between respondents. Current available guidelines recommend the use of PCC for the reversal of vitamin K-dependent oral anticoagulants in bleeding trauma patients and this was the most commonly reported product used by the respondents. ${ }^{1} 19{ }^{20} \mathrm{It}$ is evident that there is a lack of consensus in the guidelines regarding the use of other products for the reversal of INR in a bleeding trauma patient.

Permissive hypotension for bleeding trauma patients is a relatively new concept in the management of bleeding and coagulopathy in major trauma patients. This approach avoids the adverse effects of early aggressive fluid resuscitation while maintaining an adequate level of tissue perfusion. ${ }^{1} \quad 6 \quad 7$ Current research recommends that good quality evidence underpinning permissive hypotension is limited and this may explain the fact that over half of the respondents would treat the hypotension of the patient in scenario one.

There are a number of potential limitations in this study. One of the inherent problems with a survey study is achieving a sufficient response rate. In this study, $65 \%$ of hospitals contacted to complete the survey were represented; therefore, non-response bias may have occurred. Similarly, there was a number of missing data fields in the completed surveys which may have introduced an element of bias. Misinterpretation of questions within the survey should be considered; however, the survey was piloted and subsequently adapted, which should have reduced this inaccuracy.

\section{CONCLUSION}

Despite the inherent potential biases in an investigation using a survey for data collection, the results of this study highlight the similarities and variation in the management strategies used in the EDs in England and Wales for the elderly, anticoagulated trauma patient. The variations in practice reported may be due to the differences evident in the available guidelines for these patients. In the absence of fully validated, national clinical guidelines, there is a risk of suboptimal care and outcomes for the elderly, anticoagulated major trauma patients. As a result of the variation in current practice reported in this study, the need for the specific inclusion of this complex, subgroup of major trauma patients, in future national guidelines has been highlighted.

Twitter Follow Antoinette Edwards at @AntoinetteE60

Acknowledgements The authors would like to thank all the respondents who took time to complete the survey.

Contributors All authors provided substantial contributions to the conception or design of the work and contributed to the interpretation of study data. CEB drafted the work and all authors contributed to its revision for important intellectual content. All authors approved the final version. All authors agree to be accountable for all aspects of the work in ensuring that questions related to the accuracy or integrity of any part of the work are appropriately investigated and resolved.

Competing interests None declared.

Ethics approval TARN approval. Section 251.

Provenance and peer review Not commissioned; externally peer reviewed.

\section{REFERENCES}

1 Spahn DR, Bouillon B, Cerny V, et al. Management of bleeding and coagulopathy following major trauma: an updated European guideline. Crit Care 2013;17:R76.

2 Davenport RA, Tai N, West A, et al. A major trauma centre is a specialty hospital not a hospital of specialties. Br J Surg 2011;97:109-17.

3 National Audit Office. Major trauma care in England. 2010. http://www.nao.org.uk/ wp-content/uploads/2010/02/0910213es.pdf

4 Brohi K, Singh J, Heron M, et al. Acute traumatic coagulopathy. J Trauma 2003:54:1127-30.

5 DeLoughery TG. Coagulation defects in trauma patients: aetiology, recognition and therapy. Crit Care Clin 2004;20:13-24.

6 Santry HP, Alam HB. Fluid resuscitation: past, present and the future. Shock 2010:33:229-41.

7 Harris T, Thomas R, Brohi K. Early fluid resuscitation in severe trauma. BMJ 2012;345:e5752.

8 Leiblich A, Mason S. Emergence management of minor head injury in anticoagulated patients. Emerg Med J 2011;28:115-18.

9 Dossett LA, Riesel JN, Griffin MR, et al. Prevalence and implication of preinjury warfarin use. Arch Surg 2011;146:565-70.

10 Lecky FE, Omar M, Bouamra 0, et al. The effect of pre-injury warfarin use on mortality rates in trauma patients: a European multicentre study. Emerg Med J 2015;32:916-20

11 Hackam DG, Kopp A, Redelmeier DA. Prognostic implications of warfarin cessation after major trauma: a population based cohort analysis. Circulation 2005;111:2250-6.

12 Malone DL, Dunne J, Tracy K, et al. Blood transfusion, independent of shock severity, is associated with worse outcome in trauma. J Trauma 2003;54:898-907.

13 National Audit Office. Major Trauma Care in England. 2010. http://www.nao.org. uk/wp-content/uploads/2010/02/0910213.pdf

14 The National Confidential Enquiry into Patient Outcome and Death. Trauma: Who cares? 2007. http://www.ncepod.org.uk/2007t.htm

15 Hanley J. Warfarin reversal. Clin Pathol 2004;57:1132-9.

16 National Institute for Health and Care Excellence. Head injury: triage, assessment, investigation and early management of head injury in children, young people and adults. CG176. 2014. http://www.nice.org.uk/guidance/CG176

17 Keeling D, Baglin T, Tait C, et al. Guidelines on oral anticoagulation with warfarin —fourth edition. Brit J Haematol 2011;154:311-24.

18 Lier $H$, Krep H, Schöchl H. Coagulation management in the treatment of multiple trauma. Anaesthesist 2009;58:1010-26.

19 Ansell J, Hirsh J, Hylek E, et al. Pharmacology and management of the Vitamin K Antagonists. American College of Chest Physicians: Evidence-Based Clinical Practice Guidelines (8th Edition). Chest 2008;133:160S-98S.

20 Baker RI, Coughlin PB, Gallus AS, et al., The Warfarin Reversal Consensus Group. Warfarin reversal: consensus guidelines, on behalf of the Australasian Society of Thrombosis and Haemostasis. Med J Australia 2004;181:492-7. 